\title{
Analytical Challenges and Strategies in FE-EPMA
}

\author{
Silvia Richter and Philippe T. Pinard
}

\section{Central Facility for Electron Microscopy, RWTH Aachen University, Aachen, Germany}

Unquestionably, since their introduction one decade ago, field emission (FE) electron microprobes have pushed the boundaries of electron probe microanalysis (EPMA) by offering new possibilities to characterize smaller features. As with conventional microprobes, the proper selection of the analytical conditions (beam energy, beam current, acquisition time, $x$-ray line) is essential to obtain an accurate and precise quantification. With FE microprobes, the new challenge is to optimize these parameters to obtain the best spatial resolution. Our previous work [1] has shown that, as each analytical condition has a different influence on the accuracy, precision and spatial resolution, a compromise must be found depending on the characterization goals. In continuation, this work aims at evaluating the influence of instrumental parameters, such as the focusing capability, beam stability and stage reproducibility, on high resolution acquisitions, and proposing strategies to perform reliable and automated measurements.

As the interaction volume decreases with lower accelerating voltage, the beam diameter becomes a critical parameter in determining the spatial resolution. The beam diameter of a JEOL JXA8530F microprobe was measured at different beam energies and currents using the technique of Joy [2] and a sample consisting of gold flakes on a carbon substrate. As shown in Figure 1, the improvement of the spatial resolution at low beam energies and high currents is limited by the focusing capabilities of the electron column. In practise, we observed no advantage of selecting a beam energy lower than $5 \mathrm{keV}$.

The beam diameter is not the only factor changing as a function of the beam energy and current, but the position of the beam on the sample is also shifting. To measure this effect, images of $\mathrm{Sn}$ spheres on a carbon substrate were taken at different beam energies and currents. The shifts were determined by cross-correlation from a reference image (Fig. 2). Significant deviations ( $>5 \mu \mathrm{m})$ were observed. If not corrected, high spatial resolution measurements where each position on the sample is analyzed under different conditions (e.g. quantification using the same overvoltage ratio or different beam currents to measure trace and major elements) would not be possible.

Shift of the beam position also occurs over time due to drift. This can be the result of several factors: temperature fluctuations, current instability in the lenses, magnetic fields, charging due to column-wall contamination or of the specimen itself, etc. To evaluate this effect, the drift at different beam energies was measured over a period of 30 minutes (Fig. 3). As a result, the drift can be of the order of 1-2 $\mu \mathrm{m}$. The drift, in combination with the stage reproducibility, limits the possibility of automating a series of high spatial resolution measurements. By performing large back and forth movements, the stage reproducibility was estimated as 4 and $0.9 \mu \mathrm{m}$ in the $\mathrm{x}$ and $\mathrm{y}$ direction, respectively. A solution for the drift and stage reproducibility is to use a tracking procedure to ensure that the measured position corresponds to the recorded one prior to the start of the automation. To improve the accuracy of the drift correction procedure, especially on low contrast samples, micro-hardness indents or FIB markers can be used (Fig. 4). Furthermore, it is advisable to adjust the dimensionality of a measurement with respect to the size of the features of interest. For example, the chance of hitting a feature is higher if a line or grid of points is used instead of a single one. 
Results evaluating other instrumental parameters, such as the beam current stability and the precision of the stage movement, will also be presented.

[1] PT Pinard and S Richter, IOP Conf. Ser.: Mater. Sci. Eng. (2014), in press.

[2] DC Joy, J. Microsc. 208 (2002), pp. 24-34.

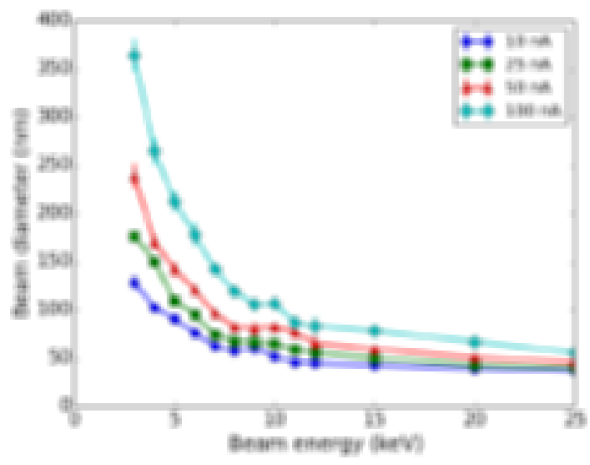

Figure 1: Measured beam diameter as a function of the beam energy and current.

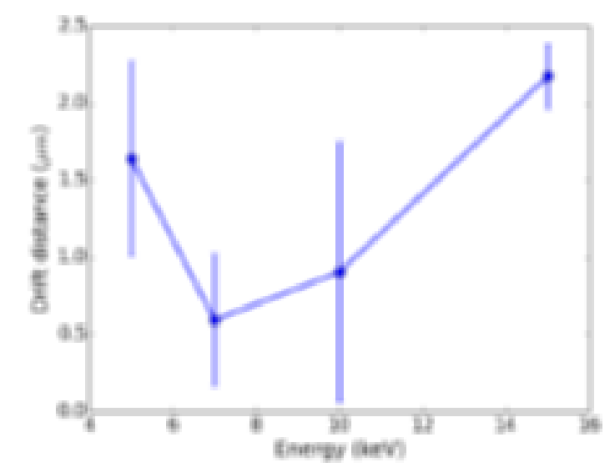

Figure 3: Maximum drift distance after $30 \mathrm{~min}$ as a function of the beam energy. 3 tests were performed at each beam energy. The beam current and magnification were kept constant at $100 \mathrm{nA}$ and 5000x, respectively.

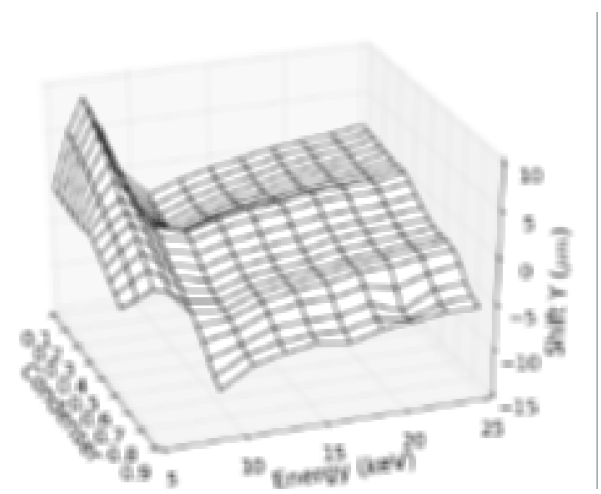

Figure 2: Shift of the beam position in the $y$ direction as a function of the beam energy and strength of the condenser lens (proportional to the beam current).

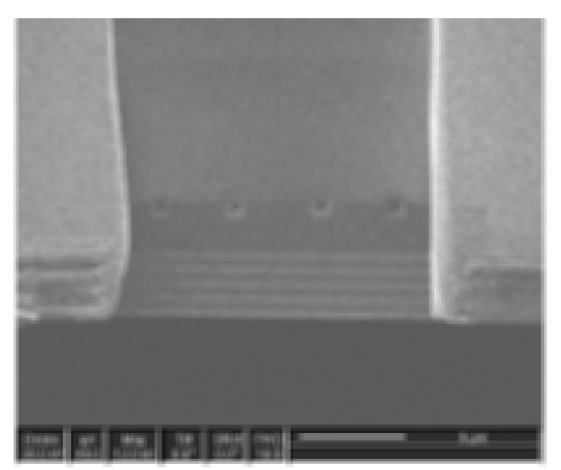

Figure 4: Focused ion beam markers (small crosses) on a $\mathrm{Ni}-\mathrm{Cr}$ multi-layered sample to improve the accuracy of the tracking procedure. 